\title{
Dynamics of Bose-Einstein condensates in cigar-shaped traps
}

\author{
A. M. Kamchatnov ${ }^{1, *}$ and V. S. Shchesnovich ${ }^{2, \dagger}$ \\ ${ }^{1}$ Institute of Spectroscopy, Russian Academy of Sciences, Troitsk 142190, Moscow Region, Russia \\ ${ }^{2}$ Instituto de Física Teórica, Universidade Estadual Paulista-UNESP, Rua Pamplona 145, 01405-900 São Paulo, Brazil
}

(Received 14 April 2004; published 12 August 2004)

\begin{abstract}
The Gross-Pitaevskii equation for a Bose-Einstein condensate confined in an elongated cigar-shaped trap is reduced to an effective system of nonlinear equations depending on only one space coordinate along the trap axis. The radial distribution of the condensate density and its radial velocity are approximated by Gaussian functions with real and imaginary exponents, respectively, with parameters depending on the axial coordinate and time. The effective one-dimensional system is applied to a description of the ground state of the condensate, to dark and bright solitons, to the sound and radial compression waves propagating in a dense condensate, and to weakly nonlinear waves in repulsive condensate. In the low-density limit our results reproduce the known formulas. In the high-density case our description of solitons goes beyond the standard approach based on the nonlinear Schrödinger equation. The dispersion relations for the sound and radial compression waves are obtained in a wide region of values of the condensate density. The Korteweg-de Vries equation for weakly nonlinear waves is derived and the existence of bright solitons on a constant background is predicted for a dense enough condensate with a repulsive interaction between the atoms.
\end{abstract}

DOI: 10.1103/PhysRevA.70.023604

PACS number(s): 03.75.Kk, 03.75.Lm

\section{INTRODUCTION}

The formation of Bose-Einstein condensates (BECs) in dilute gases [1-3] has created a field of nonlinear physics with specific problems. Among the features of BEC in gases are the spatial inhomogeneity created by the confining potential, the interplay of coherent and nonlinear phenomena, multispecies condensation, etc. Many of such problems have already been addressed in the current literature. In particular, the condensate oscillations near the center of the trap and their free expansion after switching off the trap potential were considered in Refs. [4-11]. The creation of strongly elongated ("cigar-shaped") traps has made it possible to generate dark $[12,13]$ and bright $[14,15]$ solitons in BEC. However, a theoretical description of the solitons proves to be not a simple problem. Indeed, the standard reduction of the Gross-Pitaevskii (GP) equation to the one-dimensional (1D) nonlinear Schrödinger (NLS) equation is justified only under the condition that the axial size of the solution (along the trap axis) be much greater than its radial size. However, the actual solitons observed in experiment $[14,15]$ have comparable axial and radial sizes. An attempt to go beyond the NLS approximation was made in Ref. [16] under the assumption that variation of the field variables in the axial direction takes place on a much longer scale than that in the radial direction. Therefore the equation with a "nonpolynomial interaction" obtained in Ref. [16] describes the ground state of BECs with repulsive interaction when the above assumption is correct but its application to "short" dark or bright solitons is not well justified.

In this paper we consider the dynamics of BECs in the cigar-shaped trap by means of a variational approach without

\footnotetext{
*Electronic address: kamch@isan.troitsk.ru

${ }^{\dagger}$ Present address: Departamento de Física, Universidade Federal de Alagoas, Maceió AL 57072-970, Brazil.
}

the assumption that the field variables change slowly along the trap axis. The radial distribution of the BEC density and its radial velocity are approximated by real and imaginary Gaussian functions, respectively, with parameters depending on the axial coordinate $z$. This approach allows us to reduce the 3D BEC Lagrangian to an effective 1D one which depends on the axial wave function $\Psi(z, t)$, the mean condensate radius $b(z, t)$, and the radial velocity potential $\alpha(z, t)$. The resulting system of equations for the above three variables, though being rather complicated, has the advantage of a dependence on only one spatial coordinate $z$. It contains as limiting cases the NLS and the "nonpolynomial interaction" approximations. The derived effective system allows one to investigate bright and dark solitons in a wide domain of their existence. In particular, the bright solitons can be considered right up to the collapse instability threshold. We also study the linear waves propagating along the condensate with repulsive interaction between the atoms. There are two types of waves: the low-frequency sound wave and the highfrequency radial compression wave. The dispersion relations for the linear waves, applicable to the high-density BEC, are obtained in the long-wavelength limit. In the next approximation of a "small but finite" amplitude of the ground-state perturbation we obtain the Korteweg-de Vries (KdV) equation for weakly dispersive and nonlinear waves propagating along the condensate. An interesting feature of this equation is that in a high-density BEC the coefficient in the dispersion term changes sign. An immediate consequence is that fast solitons of small amplitude, propagating in a repulsive BEC, are bright rather than dark, contrary to what is usually assumed by analogy between the GP and 1D NLS equations.

\section{GENERAL QUASI-1D EQUATIONS FOR BEC EVOLUTION}

The starting point for our consideration is the GP equation for the wave function $\psi$ of the condensate: 


$$
i \hbar \psi_{t}=-\frac{\hbar^{2}}{2 m} \Delta \psi+\frac{1}{2} m \omega_{\perp}^{2} r^{2} \psi+V(z) \psi+g|\psi|^{2} \psi,
$$

where $r=\sqrt{x^{2}+y^{2}}$ is the radial coordinate, $g=4 \pi \hbar^{2} a_{s} / m$ is the effective nonlinear coupling constant, $a_{s}$ denotes the $s$-wave scattering length, $m$ is the atomic mass, $\omega_{\perp}$ is the frequency of atomic oscillations in the radial direction, and $V(z)$ is the trap potential in the axial direction of a cigar-shaped trap. The condensate wave function $\psi$ is normalized to the number $N$ of atoms:

$$
\int|\psi|^{2} d \mathbf{r}=N
$$

The GP equation admits the least action principle formulation with the action functional

$$
S=\int L d t, \quad L=\int \mathcal{L} 2 \pi r d r d z,
$$

written under assumption that the condensate motion is axially symmetric, where the Lagrangian density is given by

$$
\begin{aligned}
\mathcal{L}= & \frac{i \hbar}{2}\left(\psi \psi_{t}^{*}-\psi^{*} \psi_{t}\right)+\frac{\hbar^{2}}{2 m}|\nabla \psi|^{2}+\frac{1}{2} m \omega_{\perp} r^{2}|\psi|^{2}+V(z)|\psi|^{2} \\
& +\frac{g}{2}|\psi|^{4} .
\end{aligned}
$$

It is well known (see, e.g., [17]) that if the linear (1D) density $n_{1}=\int_{0}^{\infty}|\psi|^{2} 2 \pi r d r$ of the condensate is small enough,

$$
\left|a_{s}\right| n_{1} \ll 1,
$$

then the 3D GP equation can be reduced to the 1D NLS equation

$$
i \hbar \Psi_{t}=-\frac{\hbar^{2}}{2 m} \Psi_{z z}+V(z) \Psi+g_{1 \mathrm{D}}|\psi|^{2} \Psi,
$$

where

$$
g_{1 \mathrm{D}}=\frac{g}{2 \pi a_{\perp}^{2}}=\frac{2 \hbar^{2} a_{s}}{m a_{\perp}^{2}}, \quad a_{\perp}=\sqrt{\frac{\hbar}{m \omega_{\perp}}} .
$$

This reduction can be performed, for example, by the variational method, when under supposition (5) the transversal degrees of freedom are "frozen" and transverse motion is reduced to the ground-state oscillations in the radial direction. As a result, the condensate wave function $\psi$ can be factorized as

$$
\psi=\frac{1}{\sqrt{\pi} a_{\perp}} \exp \left(-\frac{r^{2}}{2 a_{\perp}^{2}}\right) \Psi(z, t) .
$$

Its substitution into Eqs. (3) and (4) and integration over radial coordinate yield an effective 1D Lagrangian and the corresponding Lagrange equation for $\Psi(z, t)$ is Eq. (6) (see, e.g., [11]). Here we want to generalize this procedure to the case when $\left|a_{s}\right| n_{1} \sim 1$ and the radial motion of the condensate must be taken into account.

Assuming quasi-1D geometry, we approximate the wave function as

$$
\psi=\frac{1}{\sqrt{\pi} b(z, t)} \exp \left(-\frac{r^{2}}{2 b^{2}(z, t)}\right) \exp \left(\frac{i}{2} \alpha(z, t) r^{2}\right) \Psi(z, t) .
$$

Here $b(z, t)$ characterizes the local radius of the condensate and $\alpha(z, t) r$ gives the local radial velocity. Substitution of Eq. (9) into Eq. (4) and the result into Eq. (3) with integration over the radial coordinate yield an effective 1D Lagrangian

$$
\begin{aligned}
\mathcal{L}_{1 \mathrm{D}}= & \frac{i \hbar}{2}\left(\Psi \Psi_{t}^{*}-\Psi^{*} \Psi_{t}\right)+\frac{\hbar^{2}}{2 m}\left|\Psi_{z}\right|^{2}+\frac{1}{2} m \omega_{\perp}^{2} b^{2}|\Psi|^{2}+V(z) \\
& \times|\Psi|^{2}+\frac{g}{4 \pi b^{2}}|\psi|^{4}+\frac{\hbar}{2} \alpha_{t} b^{2}|\psi|^{2}+\frac{\hbar^{2}}{2 m}\left[\left(\frac{1}{b^{2}}+\alpha^{2} b^{2}+\frac{b_{z}^{2}}{b^{2}}\right.\right. \\
& \left.\left.+\frac{1}{2} \alpha_{z}^{2} b^{4}\right)|\psi|^{2}+\frac{i}{2} \alpha_{z} b^{2}\left(\Psi \Psi_{z}^{*}-\Psi^{*} \Psi_{z}\right)\right] .
\end{aligned}
$$

To simplify the notation, let us introduce the dimensionless variables

$$
\begin{gathered}
\tau=\omega_{\perp} t, \quad z=a_{\perp} \zeta, \quad \Psi=\frac{u}{\sqrt{a_{\perp}}}, \quad b=a_{\perp} w, \quad \alpha=\frac{\beta}{a_{\perp}^{2}}, \\
G=\frac{a_{s}}{a_{\perp}} .
\end{gathered}
$$

In the dimensionless variables the 1D Lagrangian becomes

$$
\begin{aligned}
\mathcal{L}_{1 \mathrm{D}}= & \frac{\hbar^{2}}{m a_{\perp}^{3}}\left[\frac{i}{2}\left(u u_{\tau}^{*}-u^{*} u_{\tau}\right)+\frac{1}{2}\left|u_{\zeta}\right|^{2}+\frac{1}{2} w^{2}|u|^{2}+V(\zeta)|u|^{2}\right. \\
& +G \frac{|u|^{4}}{w^{2}}+\frac{1}{2} \beta_{\tau} w^{2}|u|^{2}+\frac{1}{2}\left(w^{-2}+\beta^{2} w^{2}+w_{\zeta}^{2} w^{-2}\right. \\
& \left.\left.+\frac{1}{2} \beta_{\zeta}^{2} w^{4}\right)|u|^{2}+\frac{i}{4} \beta_{\zeta} w^{2}\left(u u_{\zeta}^{*}-u^{*} u_{\zeta}\right)\right] .
\end{aligned}
$$

Thus, in the above reduction, the evolution of BEC is described by the complex longitudinal wave function $u(\zeta, \tau)$ and two real functions, $w(\zeta, \tau)$ and $\beta(\zeta, \tau)$, corresponding to the local mean radius of the condensate and its radial velocity, respectively. Equations governing the evolution of these variables are to be obtained from the action principle

$$
S=\int L d t=\min , \quad L=\int \mathcal{L}_{1 \mathrm{D}} d \zeta
$$

with the effective Lagrangian density given by Eq. (12). We have

$$
\begin{aligned}
& i u_{\tau}+\frac{1}{2} u_{\zeta \zeta}-V(\zeta) u-3 G \frac{|u|^{2} u}{w^{2}}-\frac{1}{2}\left[\frac{2}{w^{2}}+\frac{w_{\zeta \zeta}}{w}+\left(\frac{w_{\zeta}}{w}\right.\right. \\
&\left.\left.-\frac{i}{2} \beta_{\zeta} w^{2}\right) \frac{\left(|u|^{2}\right)_{\zeta}}{|u|^{2}}-\frac{1}{2} \beta_{\zeta}^{2} w^{4}-\frac{i}{2} \beta_{\zeta \zeta} w^{2}-i \beta_{\zeta} w w_{\zeta}\right]=0 \\
&\left(w^{2}|u|^{2}\right)_{\tau}+\left[\frac{i}{2} w^{2}\left(u u_{\zeta}^{*}-u^{*} u_{\zeta}\right)+w^{4}|u|^{2} \beta_{\zeta}\right]=2 \beta w^{2}|u|^{2},
\end{aligned}
$$




$$
\begin{aligned}
\beta_{\tau}= & \frac{w_{\zeta \zeta}}{w^{3}}-\frac{w_{\zeta}^{2}}{w^{4}}+\frac{w_{\zeta}}{w^{3}} \frac{\left(|u|^{2}\right)_{\zeta}}{|u|^{2}}+\frac{1}{w^{4}}-1-\beta^{2}-\beta_{\zeta}^{2} w^{2} \\
& +\frac{i\left(u_{\zeta} u^{*}-u u_{\zeta}^{*}\right)}{2|u|^{2}} \beta_{\zeta}+2 G \frac{|u|^{2}}{w^{4}},
\end{aligned}
$$

where the longitudinal wave function is normalized as follows:

$$
\int|u|^{2} d \zeta=N
$$

Thus, we have transformed the 3D GP equation to a quasi1D form for the case of elongated cigar-shaped geometry when the radial distributions of the condensate density and its radial velocity can be approximated by simple Gaussian functions. The variables in Eqs. (14)-(16) depend only on one spatial coordinate-a strong advantage in numerical simulations. Besides that, the system (14)-(16) can be analyzed analytically in some important limiting cases (see below).

For some applications it is convenient to cast Eqs. (14)-(16) into a hydrodynamiclike form by means of the substitution

$$
u=\sqrt{\rho} \exp \left(i \int^{\zeta} v\left(\zeta^{\prime}, \tau\right) d \zeta^{\prime}\right),
$$

where $\rho(\zeta, \tau)$ is the density of BEC and $v(\zeta, \tau)$ denotes its axial velocity. As a result, we obtain the hydrodynamic system:

$$
\begin{gathered}
\rho_{\tau}+\left(\rho\left(v+\frac{1}{2} w^{2} \beta_{\zeta}\right)\right)_{\zeta}=0 \\
v_{\tau}+v v_{\zeta}+V^{\prime}(\zeta)+3 G\left(\frac{\rho}{w^{2}}\right)_{\zeta}+\frac{1}{2}\left(\frac{\rho_{\zeta}^{2}}{4 \rho^{2}}-\frac{\rho_{\zeta \zeta}}{2 \rho}+\frac{2}{w^{2}}+\frac{w_{\zeta \zeta}}{w}\right. \\
\left.+\frac{w_{\zeta} \rho_{\zeta}}{w \rho}-\frac{1}{2} \beta_{\zeta}^{2} w^{4}\right)_{\zeta}=0 \\
\beta_{\tau}=\frac{w_{\zeta \zeta}}{w^{3}}-\frac{w_{\zeta}^{2}}{w^{4}}+\frac{w_{\zeta} \rho_{\zeta}}{w^{3} \rho}+\frac{1}{w^{4}}-1-\beta^{2}-\beta_{\zeta}^{2} w^{2}+v \beta_{\zeta}+2 G \frac{\rho}{w^{4}}, \\
\left(w^{2} \rho\right)_{\tau}+\left(w^{2} \rho v+w^{4} \rho \beta_{\zeta}\right)_{\zeta}=2 \beta w^{2} \rho .
\end{gathered}
$$

In the following sections we apply the general quasi-1D equations to some important particular cases.

\section{STATIONARY SOLUTIONS}

In a stationary state all velocities are equal to zero $(v$ $=0, \beta=0$ ), the density $\rho$ does not depend on $\tau$, and the condensate wave function $u(\zeta, \tau)$ depends on $\tau$ only through the phase factor $\exp (-i \mu \tau)$, where $\mu$ is dimensionless chemical potential. Setting $\beta=0$ and introducing the stationary variables $U(\zeta)$ and $\sigma(\zeta)$ by the formulas

$$
u(\zeta, \tau)=e^{-i \mu \tau} U(\zeta), w^{2}(\zeta, \tau)=1 / \sigma(\zeta),
$$

after simple algebra we obtain from Eqs. (14)-(16) the system

$$
U_{\zeta \zeta}+\left(2 \mu-2 V(\zeta)-\sigma-\frac{1}{\sigma}-\frac{\sigma_{\zeta}^{2}}{4 \sigma^{2}}\right) U-4 G \sigma U^{3}=0,
$$

$$
\frac{1}{2}\left(\frac{\sigma_{\zeta} U^{2}}{\sigma}\right)_{\zeta}-\left(\sigma-\frac{1}{\sigma}\right) U^{2}-2 G \sigma U^{4}=0 .
$$

The system (24) and (25) has the energy functional

$$
\begin{aligned}
\mathcal{E}= & \int\left[\frac{1}{2} U_{\zeta}^{2}+V(\zeta) U^{2}+G \sigma U^{4}+\frac{1}{2}\left(\sigma+\frac{1}{\sigma}+\frac{\sigma_{\zeta}^{2}}{4 \sigma^{2}}\right) U^{2}\right. \\
& \left.-\mu U^{2}\right] d \zeta .
\end{aligned}
$$

Here the integrand can be considered as a Lagrangian with the spatial variable $\zeta$ playing the role of time. It is worth noticing that if we introduce instead of $\sigma$ an angle $\theta$ by the relation

$$
\sigma=\exp (2 \theta),
$$

then the energy functional takes the form

$$
\begin{aligned}
\mathcal{E}= & \int\left[\frac{1}{2}\left(U_{\zeta}^{2}+U^{2} \theta_{\zeta}^{2}\right)+[V(\zeta)+\cosh 2 \theta-\mu)\right] U^{2} \\
& +G e^{2 \theta} U^{4} d \zeta,
\end{aligned}
$$

which is formally an action functional of a particle moving on the plane, where the couple $(U, \theta)$ assumes the role of the polar coordinates and the external potential is

$$
\Phi(U, \theta)=[\mu-V(\zeta)-\cosh 2 \theta] U^{2}-G e^{2 \theta} U^{4} .
$$

\section{A. Small-density limit}

First of all let us consider the limit of a low density BEC:

$$
|G| U^{2} \ll 1 \text {. }
$$

In the dimensional variables, with account of the estimate $U^{2} \sim|\Psi|^{2} a_{\perp} \sim n_{1} a_{\perp}$ (where $n_{1}$ is the longitudinal density of the condensate), condition (30) coincides with Eq. (5). Since the characteristic length in the axial direction cannot be less than unity (i.e., $a_{\perp}$ in dimensional units), we find in this limit from Eq. (25):

$$
\sigma=1+\sigma_{1}, \quad\left|\sigma_{1}\right| \sim|G| U^{2} \ll 1 .
$$

Taking the estimate (31) into account, we reduce Eq. (24) to the standard (stationary) 1D NLS equation

$$
U_{\zeta \zeta}+2[\mu-1-V(\zeta)] U-4 G U^{3}=0 .
$$

This equation has been studied very well and we shall not consider it any further.

\section{B. Ground state of BEC with repulsive interaction $(G>0)$}

Another important limiting case corresponds to very slow dependence of BEC parameters on the axial coordinate $\zeta$ so 
that one can neglect the derivatives in Eqs. (24) and (25). If we denote by $Z$ the characteristic axial length, then the condition for neglecting the spatial derivatives can be written as

$$
Z^{-2} \ll G \sigma U^{2}
$$

or, in dimensional units, with $Z \sim l / a_{\perp}, G=a_{s} / a_{\perp}, \quad U^{2}$ $\sim n_{1} a_{\perp} \sim N a_{\perp} / l, \sigma \sim 1$, as

$$
\frac{a_{\perp}^{2}}{l^{2}} \ll \frac{a N}{l} .
$$

If condition (34) is satisfied, then Eq. (25) gives the relation

$$
\sigma=\left(1+2 G U^{2}\right)^{-1 / 2},
$$

equivalent to one obtained in Ref. [16]. Substitution of Eq. (35) into Eq. (24) and dropping off the derivatives relates the field $U$ to the external trap:

$$
\mu-V(\zeta)=\frac{1+3 G U^{2}}{\sqrt{1+2 G U^{2}}} .
$$

The solution of this equation with respect to $U^{2}$ yields the condensate density as a function of the axial coordinate:

$$
\rho(\zeta)=U^{2}=\frac{1}{2 G}\left\{\frac{1}{9}\left\{\mu-V(\zeta)+\sqrt{[\mu-V(\zeta)]^{2}+3}\right\}^{2}-1\right\} .
$$

This formula corresponds to the well-known Thomas-Fermi approximation for the condensate density distribution. The density $\rho$ vanishes at $\zeta=Z_{m}$ where the value of $Z_{m}$ is determined by the equation

$$
\mu-V\left(Z_{m}\right)=1
$$

which gives the relationship between $\mu$ and $Z_{m}$. The complementary relation between $Z_{m}$ and $\mu$ is given by the condition

$$
\int \rho(\zeta) d \zeta=N
$$

where the integration is taken over region where $\rho \geqslant 0$. These two relations allow us to express $\mu$ and $Z_{m}$ as functions of the number of atoms, $N$.

In the low-density limit-i.e., $G U^{2} \ll 1-$ Eq. (37) reduces to the usual formula

$$
\rho \cong \frac{1}{2 G}\left[\mu^{\prime}-V(\zeta)\right], \quad \mu^{\prime}=\mu-1,
$$

which also follows from the 1D NLS equation (32) of the preceding subsection.

For the parabolic potential

$$
V(\zeta)=\frac{1}{2} \lambda^{2} \zeta^{2}, \quad \lambda=\frac{\omega_{z}}{\omega_{\perp}},
$$

from Eq. (38) we obtain $\mu^{\prime}=\frac{1}{2} \lambda^{2} \zeta_{m}^{2}$ so that Eq. (40) with account of Eq. (39) yields the well-known expression for density distribution in the Thomas-Fermi approximation:

$$
\rho(\zeta)=U^{2}=\frac{\lambda^{2} \zeta_{m}^{2}}{4 G}\left(1-\frac{\zeta^{2}}{\zeta_{m}^{2}}\right), \quad \zeta_{m}=\left(3 G N \lambda^{-2}\right)^{1 / 3} .
$$

\section{Dark solitons in BEC with repulsive interaction}

It is well known that the NLS equation (32) with $V(\zeta)$ $=0$ and $G>0$ has a dark soliton solution

$$
U(\zeta)=U_{0} \tanh \left(U_{0} \sqrt{2 G} \zeta\right)
$$

under condition (30)—that is, $\mu^{\prime} \sim G U_{\Omega}^{2} \ll 1$. The soliton width is much greater than unity, $1 /\left(U_{0} \sqrt{G}\right) \gg 1$, and it must be much less than the longitudinal size $\zeta_{m}$ of the condensate estimated in the preceding subsection. For BEC confined by a parabolic trap we have $U_{0}^{3} \sim N / \zeta_{m}$ so that the formulated condition reads

$$
\frac{1}{\zeta_{m}^{2}} \ll \frac{G N}{\zeta_{m}} \ll 1,
$$

where the left inequality actually coincides with condition (33) in the small-density limit and the right inequality is equivalent to Eq. (30). Substitution of $\zeta_{m}$ from Eq. (42) yields the applicability condition of the soliton solution (43) in the form

$$
\sqrt{\lambda} \ll G N \ll \lambda^{-1}
$$

For large enough $N$ the right inequality in Eq. (45) is violated and we have to return to the system (24), (25). Since the left inequality in Eq. (45) is easily fulfilled for a dense BEC in a cigar-shaped trap, we suppose that the longitudinal size $\sim \zeta_{m}$ of the whole condensate is much greater than the soliton width. To find the soliton solution we take $V(\zeta)=0$ and require that $U$ satisfies the following asymptotic boundary conditions:

$$
|U| \rightarrow U_{0} \quad \text { at } \quad \zeta \rightarrow \pm \infty .
$$

Then Eq. (25) [see also Eq. (35)] gives

$$
\sigma \rightarrow \sigma_{0}=\left(1+2 G U_{0}^{2}\right)^{-1 / 2} \text { as } \zeta \rightarrow \pm \infty,
$$

while from Eq. (24) [see Eq. (36)] we find the value of $\mu$ :

$$
\mu=\frac{1+3 G U_{0}^{2}}{\sqrt{1+2 G U_{0}^{2}}}
$$

Thus we arrive at the system

$$
\begin{gathered}
U_{\zeta \zeta}+\left(2 \mu-\sigma-\frac{1}{\sigma}-\frac{\sigma_{\zeta}^{2}}{4 \sigma^{2}}\right) U-4 G \sigma U^{3}=0, \\
\frac{1}{2}\left(\frac{\sigma_{\zeta} U^{2}}{\sigma}\right)_{\zeta}-\left(\sigma-\frac{1}{\sigma}\right) U^{2}-2 G \sigma U^{4}=0,
\end{gathered}
$$

whose solution minimizes the energy

$$
\mathcal{E}=\int\left[\frac{1}{2} U_{\zeta}^{2}+G \sigma U^{4}+\frac{1}{2}\left(\sigma+\frac{1}{\sigma}+\frac{\sigma_{\zeta}^{2}}{4 \sigma^{2}}\right) U^{2}-\mu U^{2}\right] d \zeta,
$$

where $\mu$ is fixed by the asymptotic value $U_{0}$ of the wave function as dictated by formula (48).

The solution of system (49), (50) was found numerically and the results are presented in Fig. 1. As we see, even for relatively small values of $U_{0}$ (scaled by $\sqrt{G}$ in the figure) the 

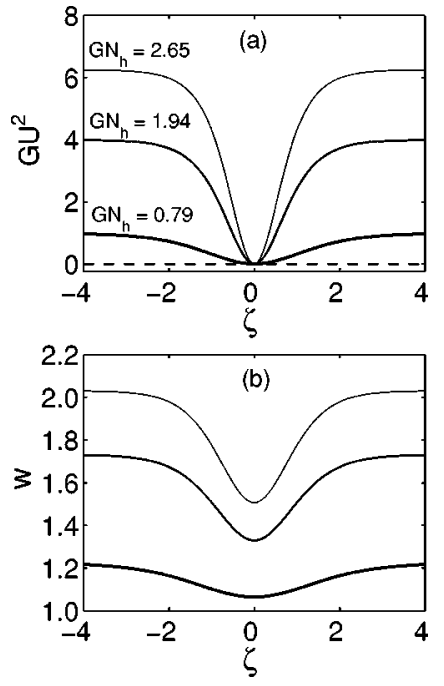

FIG. 1. The density of the dark soliton solution (a) and its transverse radius (b) vs the axial distance $\zeta$ for several values of the number of atoms in the soliton "hole."

transverse radius of the density distribution changes quite considerably along the trap axis rather than being constant as is supposed in the NLS equation approximation. The soliton width is about unity (in the dimensionless units) for our numerical solutions which also contradicts the condition (44) of applicability of the NLS equation.

The discrepancy between the improved quasi-1D description and the NLS solution can be manifested by comparison of the number of BEC atoms expelled from the dark soliton "hole," defined as

$$
N_{\mathrm{h}}=\int\left(U_{0}^{2}-U^{2}\right) d \zeta
$$

In Fig. 2 we give the plot of the number of expelled atoms versus $G U_{0}^{2}$. It shows very convincingly that the NLS approximation is quantitatively incorrect outside the region $G U_{0}^{2} \ll 1$.

We conclude this section by noticing that our quasi-1D theory permits one to describe dark solitons in the high-

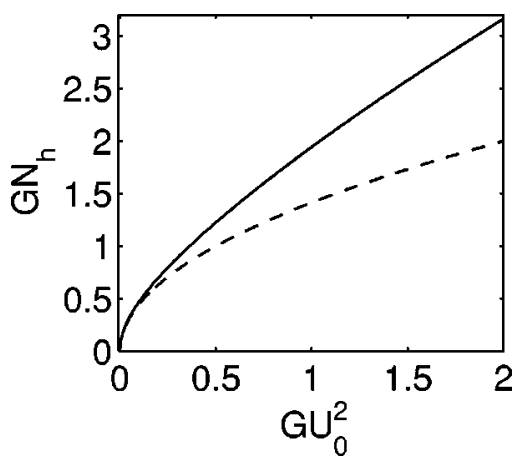

FIG. 2. The number of atoms in the dark soliton "hole" as a function of the asymptotic value $G U_{0}^{2}$. The solid line corresponds to our quasi-1D approach and the dashed line to the NLS approximation. density BEC when the standard NLS approximation breaks down.

\section{Bright solitons in BEC with attractive interaction $(G<0)$}

In the small-density limit (30) the condensate with attractive interaction between the atoms is also described by the NLS equation (32), now with $G<0$. In this case BECs can be confined in the axial direction of the cigar-shaped trap by the interatomic interaction only. Supposing the latter, we can omit the longitudinal trap potential $V(\zeta)$ and obtain the bright soliton solution

$$
U(\zeta)=\frac{U_{0}}{\cosh \left(U_{0} \sqrt{2|G|}\right)},
$$

where $U_{0}$ is the amplitude of the soliton connected with the number of atoms $N=\int U^{2} d \zeta$ by the relation

$$
N=U_{0} \sqrt{\frac{2}{|G|}},
$$

and $\mu^{\prime}=\mu-1=-|G| U_{0}^{2}$. Then condition (30) of applicability of the NLS equation can be written in the form

$$
\left|\mu^{\prime}\right|=|G| U_{0}^{2} \sim(|G| N)^{2} \ll 1 .
$$

It is clear that this condition breaks down for large enough number of atoms $N \sim 1 /|G|$ and one cannot neglect the effect of the atomic interaction on the transverse size of BEC. The transverse degrees of freedom lead to the collapse instability of BEC (see, e.g., [18]), which, as is known, also takes place in BEC confined in a cigar-shaped trap. Numerical results of Ref. [19] indicate that BEC in the cigar-shaped trap collapses for

$$
|G| N>0.676
$$

and in this region of parameters the soliton solution ceases to exist. Our quasi-1D approach captures this essential property of the attractive BEC, and hence, in our approach, the bright soliton solution can be studied in the whole region of its existence.

We have solved numerically the system (49), (50) with $G<0$ under the boundary conditions

$$
U(\zeta) \rightarrow 0 \quad \text { at } \quad \zeta \rightarrow \pm \infty .
$$

In Fig. 3 we show the wave function amplitude and transverse radius profiles of BEC for several values of $|G| N$. It is clearly seen that the amplitude of the soliton increases while its transverse radius decreases with growth of $|G| N$. For small $|G| N$ the solution is well approximated by the NLS solution (53), especially the amplitude profile. At $\zeta \rightarrow \pm \infty$ the radius approaches 1, which corresponds to the smallamplitude limit when the transverse wave function is given by the oscillator ground state. Fast decrease of $w$ in the center of the soliton with growth of $|G| N$ precedes the collapse instability of BEC.

To clarify the transition to collapse, in Fig. 4 we show the dependence of $\mu$ on $|G| N$ in our quasi-1D approximation (the solid line) and in the NLS equation case (the dashed line). We see that $\partial N / \partial \mu$ vanishes at 

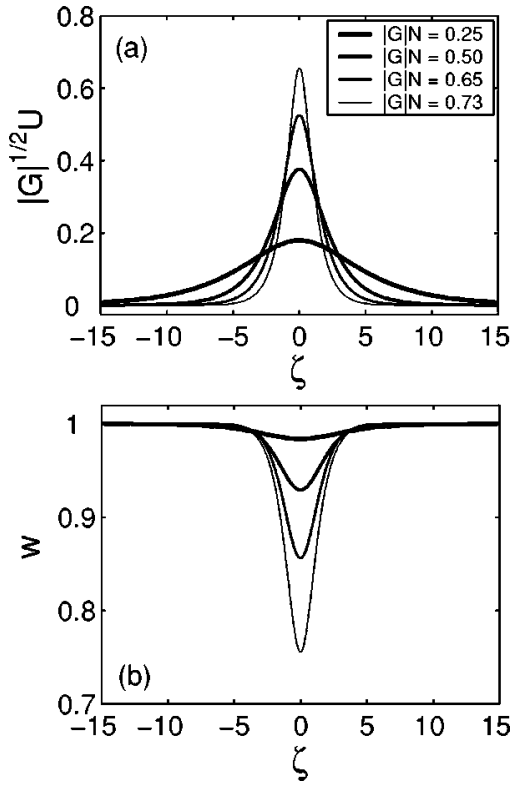

FIG. 3. The wave function amplitude (a) and transverse radius (b) of BECs corresponding to the bright soliton solution vs $\zeta$ for several values of $|G| N$.

$$
|G| N \cong 0.73
$$

which is in reasonably good agreement with the exact 3D critical value given by Eq. (56).

Finally, the axial width of our soliton solution is about unity (i.e., $\sim a_{\perp}$ in the dimensional units) in qualitative agreement with the features of bright solitons observed experimentally in Refs. [14,15].

\section{LINEAR WAVES IN CONDENSATE WITH REPULSIVE INTERACTION}

Now let us consider the linear waves propagating along a cylindrical condensate; that is, we assume that the wavelength is much less than the axial size of the condensate so that the influence of the axial trap can be neglected.

We shall start from equations in the hydrodynamical form (19)-(22) with $V^{\prime}(\zeta)=0$ in Eq. (20). In the stationary state the condensate has constant linear density $\rho_{0}$ and the transverse radius $w_{0}$ related with $\rho_{0}$ by the equation [see Eq. (35)]

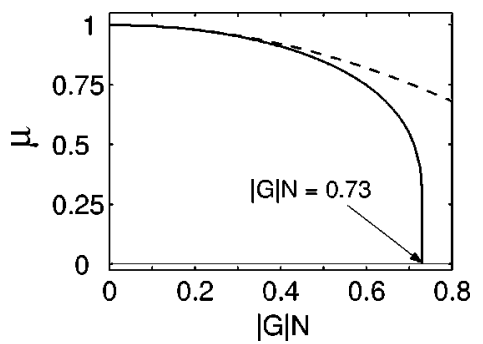

FIG. 4. The dependence of $\mu$ on $|G| N$ in quasi-1D approximation (solid line) and in the NLS equation case (dashed line).

$$
w_{0}^{4}=1+2 G \rho_{0} .
$$

In the presence of a linear wave $\rho$ and $w$ are slightly deviated from these stationary values, and a wave can be described by the small variables

$$
\rho^{\prime}=\rho-\rho_{0}, \quad w^{\prime}=w-w_{0}, \quad v, \quad \beta .
$$

Linearization of the system (19)-(22) with respect to these small variables leads to the equations

$$
\begin{gathered}
\rho_{\tau}^{\prime}+\rho_{0} v_{\zeta}+\frac{1}{2} \rho_{0} w_{0}^{2} \beta_{\zeta \zeta}=0 \\
v_{\tau}+\frac{3 G}{w_{0}^{2}} \rho_{\zeta}^{\prime}-2\left(w_{0}+\frac{G \rho_{0}}{w_{0}^{3}}\right) w_{\zeta}^{\prime}-\frac{1}{4 \rho_{0}} \rho_{\zeta \zeta \zeta}^{\prime}+\frac{1}{2 w_{0}} w_{\zeta \zeta \zeta}^{\prime}=0 \\
\beta_{\tau}-\frac{2 G}{w_{0}^{4}} \rho^{\prime}+\frac{4}{w_{0}} w^{\prime}-\frac{1}{w_{0}^{3}} w_{\zeta \zeta}^{\prime}=0 \\
w_{\tau}^{\prime}-w_{0} \beta+\frac{1}{4} w_{0}^{3} \beta_{\zeta \zeta}=0
\end{gathered}
$$

We look for a solution of the form $\left(\rho^{\prime}, w^{\prime}, v, \beta\right)$ $\propto \exp [i(K \zeta-\Omega \tau)]$, the most interesting limiting cases of which can easily be investigated. Indeed, in the longwavelength limit $K \ll 1$ we can neglect the terms with higher derivatives. Then Eq. (64) gives $\beta=w_{\tau}^{\prime} / w_{0}$ and the substitution of this into Eq. (63) yields the equation

$$
w_{\tau \tau}^{\prime}-\frac{2 G}{w_{0}^{3}} \rho^{\prime}+4 w^{\prime}=0 .
$$

For low-frequency sound wave $\Omega^{2} \ll 4$ and we obtain $w^{\prime}$ $=\left(G / 2 w_{0}^{3}\right) \rho^{\prime}$. Then after elimination of the radial variables $\beta$ and $w^{\prime}$ the system (61)-(63) reduces to a couple of equations for the longitudinal variables:

$$
\rho_{\tau}^{\prime}+\rho_{0} v_{\zeta}=0, \quad v_{\tau}+\frac{G}{w_{0}^{2}} \frac{2+3 G \rho_{0}}{1+2 G \rho_{0}} \rho_{\zeta}^{\prime}=0 .
$$

These lead at once to the expression for the sound velocity:

$$
c_{s}=\frac{\Omega}{K}, \quad c_{s}^{2}=\frac{G \rho_{0}\left(2+3 G \rho_{0}\right)}{\left(1+2 G \rho_{0}\right)^{3 / 2}} .
$$

In dimensional units we have

$$
G \rho_{0}=a_{s} w_{0}^{2} n_{1}
$$

where

$$
n_{1}=\pi a_{\perp}^{2} n
$$

is the one-dimensional density in the limit of tight radial trapping $\left(G \rho_{0} \ll 1\right)$ and $n$ is the three-dimensional density evaluated in the "center of the trap" at $r=0$. Thus $w_{0}$ is obtained from the equation $w_{0}^{4}=1+2 a_{s} w_{0}^{2} n_{1}$ which yields

$$
w_{0}=\left[\sqrt{1+\left(a_{s} n_{1}\right)^{2}}+a_{s} n_{1}\right]^{1 / 2} \text {. }
$$

Then, in dimensional units, we obtain the following expression for the velocity of sound propagating along the trap: 


$$
\widetilde{c}_{s}^{2}=\left(\frac{\hbar}{m a_{\perp}}\right)^{2} a_{s} n_{1} \frac{2+3 G \rho_{0}}{1+G \rho_{0}} .
$$

In the low-density (tight radial trapping) limit we have $G \rho_{0}$ $\sim a_{s} n_{1} \ll 1$, so that formula (71) reproduces the well-known result (see, e.g., Refs. [18,20])

$$
\tilde{c}_{s}=\frac{\hbar}{m a_{\perp}} \sqrt{2 a_{s} n_{1}} .
$$

Expression (71) gives dependence of the sound velocity on the density $n_{1}$ correct up to $G \rho_{0} \sim 1$.

Now, if we neglect all $\zeta$ derivatives in Eqs. (61)-(63), then we obtain $\rho^{\prime}=0, v=0$; i.e., there is no motion of the gas along the trap, and Eq. (65) leads to the frequency

$$
\Omega=2
$$

of compressional oscillations in radial direction. This formula agrees with the general statement $[18,21,22]$ that radial oscillations of a very elongated condensate has the frequency $\Omega=2 \omega_{\perp}$ (in dimensional units) independent of interatomic interaction.

Equations (61)-(63) permit us to find corrections to the frequencies in Eqs. (67) and (73). We have the following dispersion relation for the sound waves:

$$
\Omega^{2}=c_{s}^{2} K^{2}+\frac{1}{4}\left[1+\frac{\left(G \rho_{0}\right)^{2}\left[1-3\left(G \rho_{0}\right)^{2}\right]}{\left(1+2 G \rho_{0}\right)^{3}}\right] K^{4} .
$$

It is worth noticing that the dispersion correction changes its sign at $G \rho_{0} \cong 3.91$ and a higher-order dispersion correction has to be taken into account at this point.

For high-frequency radial compression wave we obtain

$$
\Omega^{2}=4+\frac{2+6 G \rho_{0}+5\left(G \rho_{0}\right)^{2}}{\left(1+2 G \rho_{0}\right)^{3 / 2}} K^{2} .
$$

Naturally, a correction term is not universal and depends on the atomic interaction.

\section{WEAKLY DISPERSIVE AND NONLINEAR WAVES IN REPULSIVE BECs}

At last, let us turn to waves of small but finite amplitude which propagate in repulsive BEC confined in an elongated cigar-shaped trap.

As we know from the preceding section, linear waves of long wavelength propagate with the sound velocity $c_{s}$. For finite wavelength we have to take into account the dispersion correction described by the second term in the dispersion relation (74). We must also take into account small correction arising due to nonlinear terms in the system (19)-(22) [with $V^{\prime}(\zeta)=0$ ]. To this end, we use the standard singular perturbation theory (see, e.g., [23]) and introduce the stretched variables

$$
T=\varepsilon^{3 / 2} \tau, \quad Z=\varepsilon^{1 / 2}\left(\zeta+c_{s} \tau\right),
$$

corresponding to the slow evolution of the wave in the reference system moving with the sound velocity $c_{s}$. Here $\varepsilon$ is a small parameter which characterizes the magnitude of a per- turbation of the ground state due to presence of the wave. Hence, we introduce the series expansions in powers of $\varepsilon$ :

$$
\begin{gathered}
\rho=\rho_{0}+\varepsilon \rho^{(1)}+\varepsilon^{2} \rho^{(2)}+\cdots, \quad w=w_{0}+\varepsilon w^{(1)}+\varepsilon^{2} w^{(2)}+\cdots, \\
v=\varepsilon v^{(1)}+\varepsilon^{2} v^{(2)}+\cdots, \quad \beta=\varepsilon^{3 / 2} \beta^{(1)}+\varepsilon^{5 / 2} \beta^{(2)}+\cdots .
\end{gathered}
$$

Substitution of these series expansions into Eqs. (19)-(22) gives, in the first two orders in powers of $\varepsilon$ of the groundstate formula (59), the relationships

$$
w^{(1)}=\frac{G}{2 w_{0}^{3}} \rho^{(1)}, \quad v^{(1)}=-\frac{c_{s}}{\rho_{0}} \rho^{(1)}, \quad \beta^{(1)}=\frac{G c_{s}}{2 w_{0}^{4}} \rho_{Z}^{(1)},
$$

and reproduces the formula (67) for the sound velocity. The last relevant terms of the expansions in powers of $\varepsilon$ yield the equations

$$
\begin{aligned}
-c_{s} \beta_{Z Z}^{(1)} & +\frac{1}{w_{0}^{3}} w_{Z Z Z}^{(1)}+\frac{20}{w_{0}^{2}} w^{(1)} w_{Z}^{(1)}-\frac{8 G}{w_{0}^{5}}\left(\rho^{(1)} w^{(1)}\right)_{Z}=\frac{4}{w_{0}} w_{Z}^{(2)} \\
& -\frac{2 G}{w_{0}^{4}} \rho_{Z}^{(2)}, \\
& \rho_{T}^{(1)}+\left(v^{(1)} \rho^{(1)}\right)_{Z}+\frac{1}{2} \rho_{0} w_{0}^{2} \beta_{Z Z}^{(1)}=-c_{s} \rho_{Z}^{(2)}-\rho_{0} v_{Z}^{(2)}, \quad \\
v_{T}^{(1)}+ & v^{(1)} v_{Z}^{(1)}+\frac{6\left(3 G \rho_{0}+1\right)}{w_{0}^{4}} w^{(1)} w_{Z}^{(1)}-\frac{6 G}{w_{0}^{3}}\left(\rho^{(1)} w^{(1)}\right)_{Z} \\
& -\frac{1}{4 \rho_{0}} \rho_{Z Z Z}^{(1)}+\frac{1}{2 w_{0}} w_{Z Z Z}^{(1)}=-c_{s} v_{Z}^{(2)}+\frac{2\left(3 G \rho_{0}+1\right)}{w_{0}^{3}} w_{Z}^{(2)} \\
& -\frac{3 G}{w_{0}^{2}} \rho_{Z}^{(2)},
\end{aligned}
$$

which can be considered as a linear system for the variables $\rho_{Z}^{(2)}, w_{Z}^{(2)}, v_{Z}^{(2)}$ [we have omitted the equation following from Eq. (22) which gives the expression for $\beta^{(2)}$ in terms of the other variables]. It is easy to find that the right-hand sides of Eqs. (79)-(81) are linearly dependent. Hence, for existence of a nontrivial solution, the left-hand sides must satisfy the corresponding compatibility condition which with account of Eqs. (78) can be transformed to the $\mathrm{KdV}$ equation taking after transition to the "laboratory" reference frame with variables $\tau, \zeta$ and density disturbance $\rho^{\prime}=\varepsilon \rho^{(1)}$ of the form

$$
\begin{gathered}
\rho_{\tau}^{\prime}-c_{s} \rho_{\zeta}^{\prime}+\frac{1}{8 c_{s}}\left[1+\frac{\left(G \rho_{0}\right)^{2}\left(1-3\left(G \rho_{0}\right)^{2}\right)}{\left(1+2 G \rho_{0}\right)^{3}}\right] \rho_{\zeta \zeta \zeta}^{\prime} \\
-\frac{3 G}{c_{s} w_{0}^{2}}\left[1+\frac{\left(G \rho_{0}\right)^{2}}{2\left(1+2 G \rho_{0}\right)^{2}}\right] \rho^{\prime} \rho_{\zeta}^{\prime}=0 .
\end{gathered}
$$

The linear terms here reproduce the first two terms (74) of the expansion of the dispersion relation in powers of $K$. This $\mathrm{KdV}$ equation has well-known soliton solutions.

In the low-density limit $G \rho_{0} \ll 1$, Eq. (82) takes the form 


$$
\rho_{\tau}^{\prime}-c_{s} \rho_{\zeta}^{\prime}+\frac{1}{8 c_{s}} \rho_{\zeta \zeta \zeta}^{\prime}-\frac{3 G}{c_{s}} \rho^{\prime} \rho_{\zeta}^{\prime}=0, \quad G \rho_{0} \ll 1 .
$$

It is worthwhile to note that this equation can be derived directly from the 1D NLS equation (6) which is a correct approximation to the GP equation in this limit. Indeed, the evolution of a small-amplitude disturbance $a(z, t)$ of the ground state $\Psi_{0}=$ const-i.e., $\Psi=\left[\Psi_{0}+a(z, t)\right] \exp [-i \omega t$ $+i \phi(z, t)]$ — is governed by the $\mathrm{KdV}$ equation (see, e.g., [24])

$$
a_{t}-\tilde{c}_{s} a_{z}+\frac{\hbar^{2}}{8 m^{2} \widetilde{c}_{s}} a_{z z z}-\frac{3 g_{1 D} \Psi_{0}}{m \widetilde{c}_{s}} a a_{z}=0,
$$

where $\widetilde{c}_{s}$ is the dimensional sound velocity (72). Then the transformation of this equation to dimensionless units (11) with account of the relationship $\rho^{\prime} \cong 2 a_{\perp} \Psi_{0} a$ between small disturbances of the density $\left(\rho^{\prime}\right)$ and the amplitude $(a)$ reproduces Eq. (83).

A remarkable feature of Eq. (82) is the fact that the dispersion term changes sign at $G \rho_{0}=3.91$ while the coefficient at the nonlinear term is always negative. This means that the small-amplitude solitons propagating on a constant background in repulsive BEC are dark only in the region $G \rho_{0}<3.91$ but become bright in the region $G \rho_{0}>3.91$. Obviously, such behavior is explained physically by competition of two mechanisms of energy changes due to presence of the wave-one is caused by the change of the potential energy of the gas in the transverse trap and another one is related with the nonlinear energy of interatomic gas interaction. Thus, we have found that bright solitons can propagate along dense enough repulsive BEC confined in a cigarshaped trap. These solitons propagate with the velocity close to the speed of sound.

\section{CONCLUSION}

The effective one-dimensional system derived in this paper describes the motion of condensate in a wide region of parameters when the radial distribution has not reached yet the Thomas-Fermi limit and the characteristic axial dimension is not less than the BEC radius in an elongated cigarshaped trap. This system depends only on one spatial coordinate, which can greatly simplify numerical simulations of nonlinear effects in BEC. The advantage of our approach is illustrated by applications to description of dark and bright solitons in the high density BEC. The one dimensionality of the effective system permits one to develop various analytical approximations. In particular, the dispersion relations for sound and radial compression waves are obtained for highdensity BEC. The evolution of small but finite amplitude perturbations is described by the $\mathrm{KdV}$ equation which leads to qualitatively new behavior of BEC at high enough density: small-amplitude bright solitons can propagate on a constant background in a repulsive BEC, whose density is above some threshold value. This prediction can be verified by experiment.

We hope that the quasi-1D theory developed in this paper will find applications in descriptions of many other nonlinear processes in the high-density BEC confined in the cigarshaped trap-i.e., when the standard one-dimensional nonlinear Schrödinger equation loses its applicability.

Note added in proof. Recently we learned that a formula equivalent to Eq. (37) was obtained in Ref. [25]. We are grateful to Professor L. Salasnich for this remark.

\section{ACKNOWLEDGMENTS}

This work was supported by FAPESP and FAPEAL/CNPq grants (Brazil). A.M.K. thanks also RFBR for partial support.
[1] M. N. Anderson, J. R. Ensher, M. R. Mathews, C. E. Wieman, and E. A. Cornell, Science 269, 198 (1995).

[2] K. B. Davis, M.-O. Mewes, M. R. Andrews, N. J. van Druten, D. S. Durfee, D. M. Kurn, and W. Ketterle, Phys. Rev. Lett. 75, 3969 (1995).

[3] C. C. Bradley, C. A. Sackett, J. J. Tollett, and R. G. Hulet, Phys. Rev. Lett. 75, 1687 (1995); 79, 1170(E) (1997);C. C. Bradley, C. A. Sackett, and R. G. Hulet, ibid. 78, 985 (1997).

[4] Y. Castin and R. Dum, Phys. Rev. Lett. 77, 5315 (1996).

[5] Yu. Kagan, G. V. Shlyapnikov, and J. T. M. Walraven, Phys. Rev. Lett. 76, 2670 (1996).

[6] Yu. Kagan, E. L. Surkov, and G. V. Shlyapnikov, Phys. Rev. A 54, R1753 (1996).

[7] F. Dalfovo, C. Minniti, S. Stringari, and L. Pitaevskii, Phys. Lett. A 227, 259 (1997).

[8] J. A. Stickney and A. A. Zozulya, Phys. Rev. A 65, 053612 (2002).

[9] L. Plaja and L. Santos, Phys. Rev. A 65, 035602 (2002).

[10] K. K. Das, Phys. Rev. A 66, 053612 (2002).

[11] A. M. Kamchatnov, Zh. Eksp. Teor. Fiz. 125, 1041 (2004), e-print cond-mat/0310550.
[12] S. Burger, K. Bongs, S. Dettmer, W. Ertmer, K. Sengstock, A. Sanpera, G. V. Shlyapnikov, and M. Lewenstein, Phys. Rev. Lett. 83, 5198 (1999).

[13] J. Denschlag, J. E. Simsarian, D. L. Feder, C. W. Clark, L. A. Collins, J. Cubizolles, L. Deng, E. W. Hagley, K. Helmerson, W. P. Reinhart, S. L. Rolston, B. I. Schneider, and W. D. Phillips, Science 287, 97 (2000).

[14] L. Khaykovich, F. Schreck, G. Ferrari, T. Bourdel, J. Cubizolles, L. D. Carr, Y. Castin, and C. Salomon, Science 296, 1290 (2002).

[15] K. E. Strecker, G. B. Partridge, A. G. Truscott, and R. G. Hulet, Nature (London) 417, 150 (2002).

[16] L. Salasnich, A. Parola, and L. Reatto, Phys. Rev. A 65, 043614 (2002).

[17] V. M. Pérez-García, H. Michinel, and H. Herrero, Phys. Rev. A 57, 3837 (1998).

[18] L. Pitaevskii and S. Stringari, Bose-Einstein Condensation (Clarendon Press, Oxford, 2003).

[19] A. Gammal, L. Tomio, and T. Frederico, Phys. Rev. A 66, 043619 (2002)

[20] E. Zaremba, Phys. Rev. A 57, 518 (1998). 
[21] Yu. Kagan, E. L. Surkov, and G. V. Shlyapnikov, Phys. Rev. A 54, R1753 (1996).

[22] L. P. Pitaevskii, Phys. Lett. A 221, 14 (1996).

[23] A. M. Kamchatnov, Nonlinear Periodic Waves and Their Modulations-An Introductory Course (World Scientific, Sin- gapore, 2000).

[24] Yu. S. Kivshar and B. Luther-Davies, Phys. Rep. 298, 81 (1998).

[25] L. Salasnich, A. Parola, and L. Reatto Phys. Rev. A 69, 045601 (2004). 